\title{
ADOPTING IMMERSIVE TECHNOLOGIES FOR DESIGN PRACTICE: THE INTERNAL AND EXTERNAL BARRIERS
}

\author{
Hoang, Dieu (1); Naderi, Ehsan (1); Cheng, Renée (1); Aryana, Bijan (2) \\ 1: University of Minnesota, United States; 2: Chalmers University of Technology
}

\begin{abstract}
In the first quarter of the new millennium, the immersive technologies such as Virtual Reality (VR), Augmented Reality (AR), and Mixed Reality (MR) are only a few steps away from becoming the mainstream tools within the design industry. This study investigated the internal and external barriers of technology adoption within design-oriented businesses. A mixed method was used to collect and analyze the data from the employees of a large design firm. This research confirmed that external barriers such as funding, technical support, training, and business strategy that exist at the organizational level are interrelated with the internal barriers such as designers' and managers' perception and attitude toward the new technologies. The managerial applications were discussed later and the directions for future research were provided.
\end{abstract}

Keywords: Virtual reality, Immersive technologies, Technology adoption, Design practice, Research methodologies and methods

\section{Contact:}

Hoang, Dieu

University of Minnesota

Masters of Science in Architecture: Research Practices

United States of America

hoang256@umn.edu 


\section{INTRODUCTION}

The concept of a virtual environment has been present for decades. In the earlier stages of development, the use of virtual environments was found most in costly flight simulation or military training. However, computer power and displays have become much more affordable over the year, Virtual Reality (VR) and Augmented Reality (AR) have been more accessible to the public (Adam, 1993). Although it has taken some time, immersive technology has been adopted in many industries, serving endless needs (Berg and Vance, 2017). Besides gaming, the biggest industry for the development of such immersive technologies, other professions that have been highly benefited from VR, AR, MR include entertainment, manufacturing (Azuma, 1997) healthcare, training, education, public policy, strategic communication (Zyda, 2005), product and human-focused designs (Pontonnier et al., 2014), and other design driven areas such as architecture (Thomas, 2012), landscape architecture and environmental planning (Portman et al., 2015). Within the design professions, especially human-centred design, it has been proven that the adoption of the immersive technologies in design has positive impacts on the design process, which allows designers and clients to have a better understanding of a project. These technologies also play a significant role as a collaboration tool that minimizes difficulties in communication and enhances the quality of the collaborative design process (Frost and Warren, 2000). The use of VR, AR, and MR is not only limited to co-located collaboration, but it is now also well utilized in remote communication and net-based use (Thomas, 2012). Many research papers have studied and proven the capabilities and potentials of VR, AR, and MR in a variety of industries, however, studies that assist a design-oriented business on how to successfully integrate immersive technologies on a management level, and how to identify and overcome barriers of adopting a new technology have not been tackled as often.

In recent years, many design firms have started to adopt these immersive technologies and partially employ them in their workflow because of the advantages that they offer to design practices. However, those design organizations that plan to adopt these new techniques must consider the concerns that come with the upgrade (Nambisan, 2002). Some components such as technological assets, organizational assets, external assets (Adler and Shenbar, 1990), and employees' learning curve (Nambisan, 2002) should be carefully considered and addressed during the adoption process in order to guarantee the success of the firm's technological base.

Technology should be thought of as an experience rather than simply technological artifacts (McCarthy and Wright, 2004). Thus, within this study, Virtual Reality, Augmented Reality, and Mixed Reality adoption does not express one knowing how to use the equipment. It rather embodies the process of learning, understanding, utilizing, and engaging with the technologies coming together to form a coherent whole of experience. By using the term "immersive" in the context of this study, we mean new visualization and presentation technologies including Virtual Reality, Augmented Reality, and Mixed Reality. Although these technologies are similar in some aspects, they also have some unique factors that make them distinct from each other. Virtual Reality (VR) is "a medium composed of interactive computer simulations that sense the participant's position and actions and replace or augment the feedback to one or more senses, giving the feeling of being mentally immersed or present in the simulation (a virtual world)" (Sherman and Craig, 2018, p.13). Augmented Reality (AR) maybe identified as a special case of VR according to Milgram. AR attempts to embed digitally developed supplements into the real environment, or into a live video of the real environment. The augmented information requires to embody a consistent link to the real world. This link is mostly a spatial relation between the augmentations and the actual environment (Milgram et al., 1995). Mixed Reality (MR) is a medium that provides an environment merging the actual and digital environments to build a new complexity of interaction, where both real-life and virtual objects co-exist and interact in real time (Di Loreto et al., 2011).

Challenges of adopting immersive technologies have been previously studied in other contexts (Rogers, 2000). In educational context for example, what have been found to stop users from adopting new technologies can be identified as internal barriers and external barriers. Internal barriers can be described as socio-cultural factors (Bereiter, 1994) such as concerns about privacy and appearance (Billinghurst, Clark, and Lee, 2015) as well as perception and attitude of users (Schieman and Fiordo, 
1990). On the other hand, external barriers consist of exposure to technology, institutional support, training time constraints and learning curve (Rogers, 2000). As a hierarchical model of adoption of technology, there comes a system of benchmarking with five levels of adoption: familiarization, utilization, integration, reorientation, and evolution (Hooper and Rieber, 1995). By default, any organization wanting to adopt emerging technologies in their practice shall go through each of these stages and strive to reach evolution, which is the final level of adoption. The following are brief descriptions of the five level of adoption of new technologies based on the study conducted by Hooper and Rieber, identifying five levels of adopting immersive technologies technology including: a) Familiarization: Light exposure to or experience with a technology. An example would be a person participating in a workshop that covers "how to's" of a technology. The person becomes acquainted with the technology. All the knowledge that remains, is a memory of the experience. b) Utilization: This stage occurs when the person starts to try out the technology in practice, and prematurely satisfied with their limited use of technology. Practitioners who progress only to this stage will most likely discard the technology when they first face a sign of issue due to the lack of commitment. c) Integration: Integration is identified as the break through phase when the practitioner consciously decides to commit to responsibilities to the technology. If the technology is suddenly removed or becomes unavailable, the person will find it hard working without it. d) Reorientation: Practitioners in this stage will be open to technologies embracing their work yet will not be threatened by being replaced by technology. They tend to reconsider and re-conceptualize their practice taking advantage of the technologies. e) Evolution: This is the final stage of technology adoption model, where practitioners continue to evolve, adapt to the ever-changing technological climate and remain effective. There will never be a final solution or conclusion, and to be searching for one means that they are missing the point.

This research project aims to take a closer look at the adoption of immersive technologies that include Virtual Reality (VR), Augmented Reality (AR) and Mixed Reality (MR) within the design area and focuses on identifying the challenges of the technology adoption process in the design practice setting. Therefore, the present study attempts to address the following research questions:

RQ1: What technology adoption barriers are applicable to the design profession?

RQ2: How does learning curve affect the technology adoption within the design industry?

To investigate the research questions, the authors conducted two studies. In the first study, the qualitative method was employed to identify the technology adoption barriers. The focus group sessions with designers revealed several obstacles for adopting immersive technologies by designers and design firms. The second quantitative study was designed and conducted to measure the learning curve of design firm employees and their attitude toward learning and using new technologies. In the following sections, the methodology and results of each study are explained. Then, discussion of results, future direction of research, as well as limitations of study are discussed.

\section{STUDY 1}

As an approach to find the response to the first research question, in the first study, a focus group session was conducted with six participants from the design firm in order to capture their perspectives on the immersive technologies adoption, and what is holding them back from using these new technologies in their regular work process.

\subsection{Methodology}

Study 1 was the qualitative part of this study, starting with a focus group in order to determine the challenges of technology adoption for design and development team. Then the collected data from this phase of research was validated by comparing and contrasting it with the technology adoption barriers found in the literature. The obtained insights from focus group defined the scope of work for the following phase of research. 
The research was conducted at a large-sized (1200 employees) international design and engineering consultancy firm in the United States. The research was particularly conducted with employees (design practitioners) of a local office in a major Midwestern metropolitan area with 80+ employees. Focus group meeting consisted of six participants, who were design practitioners at the design firm. Participants of the focus group were deliberately chosen from the pool of employees in order to cover different aspects of the issue and bring a variety of perspectives based on their experience and positions within the firm. The group consisted of senior designers, junior designers, and project managers. The focus group meeting was held at one of the design firm's conference rooms. During the focus group session, an unbiased moderator facilitated the discussion among the participants and an observer took notes for further analysis. The whole session was audio recorded and transcribed --with participants' consent-- without identifier for anonymous investigation. The topics of discussion were not specific to any kind of technology. They only focused on participant's attitudes and concerns towards the integration of VR, AR, and MR in the design process in the future. The discussion started with the unbiased moderator asking open ended questions to the participants regarding their experience and current knowledge relating to the adoption of immersive technologies within their design firm. For example, the following open-ended question was used to facilitate the focus group discussion: "what is your take on your firm's progress of adopting VR, AR, and MR in the design service?". The discussion was then carried on with a conversation among the participants themselves, without any interruption from the moderator, discussing their opinions on the immersive technologies adoption process over all, and what was challenging them within the process.

\subsection{Analysis and results}

The technology adoption barriers which were identified in the focus group sessions were divided into two internal and external categories (Roger, 2000) (See Table 1).

There were three items identified as internal barriers. According to focus group participants, the perceptions of the use of immersive technologies could be challenging. Many designers within the firm believed that VR, AR, and MR could be useful on finished designs and marketing, while they did not see how the technologies could be effectively integrated in the design process. The attitude toward the integration of these technologies in the firm's design service was debated among the participants. Most participants believed that every extra design-related activity that required the use of VR/AR/MR should have been added to the project expenses and eventually should be reflected on the design service quote and bill. Firm managers seemed to think of VR, AR, and MR as a product, technological artifacts, or a marketing tool rather than a production technology, which directly impacts the process of design. According to Ford (1988), any technology evolution within a corporate firm takes 5-10 years to achieve. This timeline surpasses any design firm's typical strategic planning, whose objectives are designed to be achieved within 3-5 years. With that mindset, many managers tend to avoid the long-term vision of technology adoption and rather see current technological products as a marketing strategy (Ford, 1988). On the other hand however, some participants believed that it was important for the designers to learn and adopt the technology to improve the quality of design. In fact, these participants believed that integration of immersive technologies in design practice would be an investment rather than a profitable service in the short-term. Although, all participants had some levels of experience with VR/AR/MR, yet they expressed their fear of learning the new technology due to the time constrain and workload.

The focus group discussion also brought up other concerns and challenges of technology adoption that could be categorized as external barriers. There was a lack of knowledge on what type of hardware and software would best fit the firm's design processes. In addition, different individuals had a different learning curve. Therefore, training sessions must be provided to designers. Identifying and funding the appropriate employee training program and purchasing reliable and upgradeable technologies could be a challenge as it is hard for many companies whose service is not technology development oriented. The leadership and managerial support could also become another barrier. Design managers often times are aware of the potential of employing the VR, AR, and MR in design processes and services, and express their excitement to adopt those technologies. However, excitement and verbal agreement is not enough to accelerate the technology adoption. It requires more pragmatic effort in the managerial level to tackle this challenge in the design firm. Finally, lack of lead users and 
early adopters in design firms was identified as the last barrier, because those early adopters would encourage others to maximize the use of VR, AR, and MR.

Table1. Technology adoption barriers according to focus group session

\begin{tabular}{|l|r|}
\hline $\begin{array}{l}\text { Internal barriers: attitudes, perceptions, } \\
\text { openness, willingness }\end{array}$ & $\begin{array}{r}\text { External barriers: availability, accessibility, } \\
\text { institutional \& technical support }\end{array}$ \\
\hline $\begin{array}{l}\text { Designer's perception of the use of } \\
\text { VR/AR/MR }\end{array}$ & Availability and reliability of hardware and software \\
\hline $\begin{array}{l}\text { Manager's attitude toward VR/AR/MR } \\
\text { integration in design service }\end{array}$ & Training program for employees \\
\hline & Funding availability for equipment, and training \\
\hline & Support from business managers \\
\hline & Role models/champions in the design firm \\
\hline & Availability of technical support \\
\hline
\end{tabular}

\section{STUDY 2}

In the second study aims to seek answer to the second research question. A survey was designed and distributed among the design firm employees in order to measure the designers' attitude toward the adoption of immersive technologies and their self- assessment of learning curve for utilization of technology with its highest standard. Overall, 54 responses were collected.

\subsection{Methodology}

Follow up survey was designed based on the obtained insight from the findings of study 1 as well as the challenges that were identified in the literature. It was distributed among the employees anonymously. The purpose of the survey was to further validate data from focus group, and most importantly to quantitatively determine employees' attitude toward the use of VR, AR, and MR and their learning curve for effective use of those tools in the production and delivery of design services. This survey was distributed to all of the firm's employees through the company's internal email platform.

In addition to demographic questions, the survey consisted of several multiple-choice questions for examining the employees' perspectives towards integrating immersive technologies such as VR, AR, and MR in their design services, exposure to the technologies and learning curve. The survey was designed to capture different nuances of VR/AR/MR utilization for each individual participant based on a rating system of five levels of utilization. Level 1 involved employees that were not using the immersive technologies in their design projects at the time of survey. They showed no interest in adopting the technologies as well as learning the technology. Level 2 represented participants who had not used the technologies yet showed interest in adoption with no willingness to learn the technologies. Level 3 were participants who had not used the technologies but showed interest in adoption and learning. Level 4 stood for those employees who had already used the VR/AR/MR technologies in their practice. These design practitioners had some levels of experience with technology but were still interested in learning from training. Level 5 included design practitioners that constantly used the immersive technologies in most of their design projects. These designers have already adopted the immersive technologies and require minimal training. The survey also measured the level of comfort of participants with using the technologies among the participants. Participants were asked to rate their level of comfort with using the immersive technologies from scale 1 to 5 (1: not comfortable at all, 5: extremely comfortable). The learning curve for adopting technology was measured using each employee's preferred way of learning, as well as the amount of time per week that they were willing to spend on learning a new technology. For example, the following is the 
question used for measuring employee's most desired way of learning: "which of the following would you prefer as your method of learning a new technology: self-teaching; online courses; one on one training; small group sessions; and large group sessions?"

\subsection{Analysis and results}

A total number of 54 participants responded to the survey which was designed to assess the attitude of design practitioners toward adopting immersive technologies as well as their self-evaluation of learning curve. Participants had different roles in the design firm including: designer, project architect, project manager, engineer, information technology support, marketer, business manager, as well as other roles such as management principles, researchers and design interns. Majority of participants were architectural designers. Among all participants, junior design positions with less than 3 years of experience was the major group of participants. This group was followed by a group of senior designers with more than 10 years of experience.

The survey results indicated that only $3.7 \%$ of participants showed a sign of a full utilization of VR/AR/MR (level 5), in their design practice. Majority of respondents (96.3\%) showed their interest in adopting immersive technologies either with or without interest in learning the technology; and finally, a small percentage of respondents (3.7\%) showed no interest in adoption and learning the technology (See Figure 1).

The results also showed that $31.7 \%$ of participants were comfortable with production of 360 rendering of still images, $28 \%$ were comfortable with creation of walk-through animation video, and after those, $21.5 \%$ with VR production, $11.2 \%$ with AR production, and $7.6 \%$ with MR production (See Figure 2).

The results for measuring the participants' learning curve showed that designers prefer to learn the new technologies in the context of small training group sessions. Also, 29.6\% of participants were interested in self-learning activities. On the other hand, less participants were interested in one-on-one learning with a mentor $(22.2 \%)$ as well as online training programs (16.7\%). The results also showed that participants were not interested in large group training sessions (See Figure 3).

Finally, participants self-reported the amount of time that they were willing to spend on learning a new VR, AR, and MR technology. Most participants (35.2\%) were willing to dedicate 2 to 4 hours/week for learning the new technologies. In the next category, $27.8 \%$ of participants were willing to spend up to 4 to 8 hours/week on learning the technology. The learning time of less than 2 hours/week $(22.2 \%)$ and more than 8 hours/week (8\%) were the least preferred options (See Figure 4).

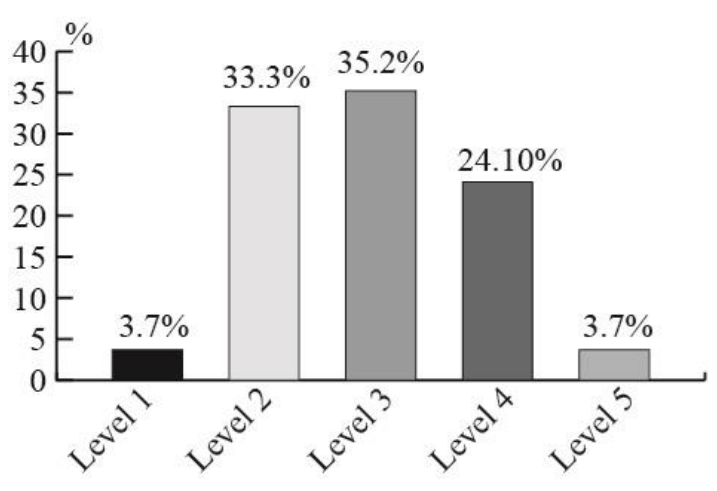

Figure 1. Level of utilization

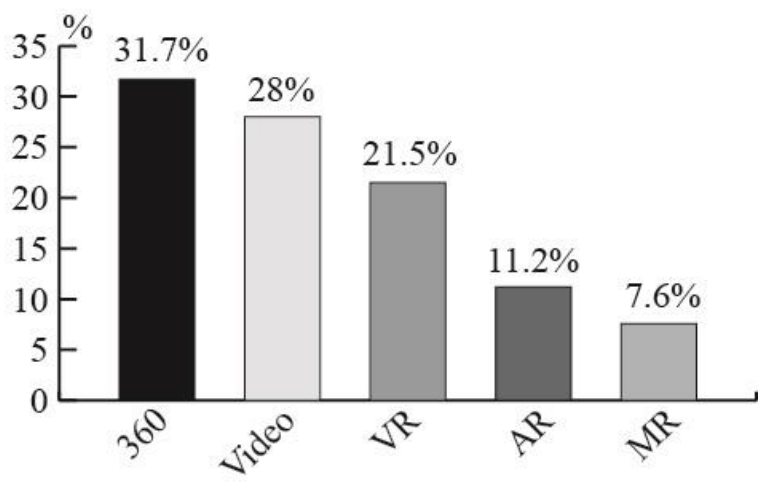

Figure 2. Comfortability with technologies 


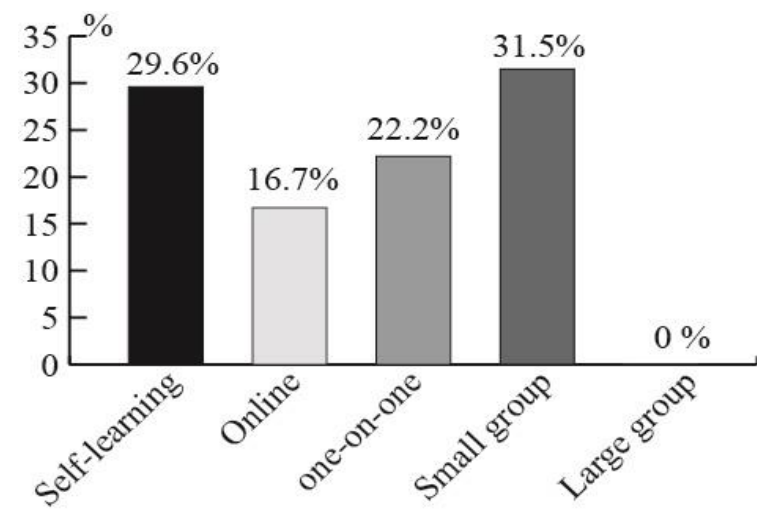

Figure 3. Learning curve: preferred training

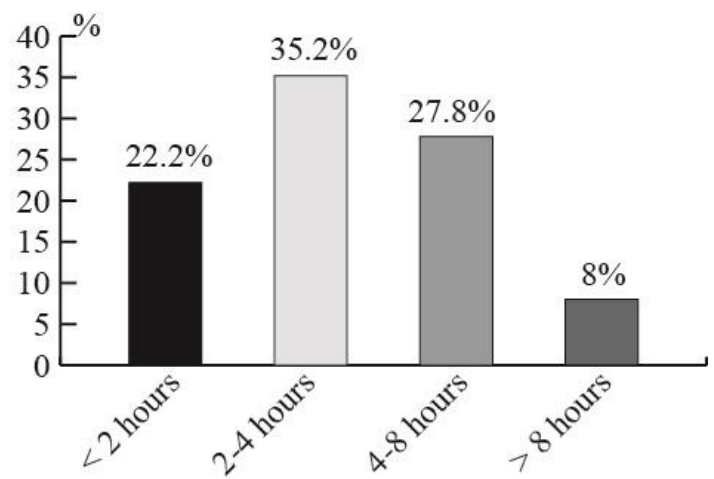

Figure 4. Learning curve: hours of learning/week

As a follow up, a standard multiple regression analysis was performed between attitude toward the adoption of emerging technologies and years of experience of employees as well as their willingness to spend time on learning emerging technologies for design services. The regression analysis revealed neither of these variables were significant predictors of attitude toward the adoption of immersive technologies $(\mathrm{F}(2,25)=0.62, \mathrm{p}=0.55$, with an $\mathrm{R} 2$ of 0.05$)$. In terms of individual relationship between the variables and attitude toward the adoption of emerging technologies, years of experience $(\mathrm{t}=0.23$, $\mathrm{p}=0.29)$, and willingness to spend time on learning emerging technologies $(\mathrm{t}=0.45, \mathrm{p}=0.65)$ were found not to be significant predictors of immersive technology adoption.

\section{DISCUSSION}

The focus group phase of the present study confirmed Roger's (2000) proposed barriers of technology adoption. In fact, all of the identified items were corresponding to five barriers of technology adoption (Roger, 2000) including familiarization, utilization, integration, reorientation, and evolution. This result implies that the barriers of technology adoption is not only applicable to a particular business practice. Indeed, technology adoption barriers are fully applied to design profession in different levels. Thus, any strategy that is found to successfully resolve any of the technology adoption barriers within a specific discipline should also be well analysed and considered to be adapted by other professions rather than being limited to only one context and setting.

The majority of the participants of this study had awareness of the potential positive values of VR, AR, and MR in their design practice. They showed different attitudes toward technology adoption in terms of interest in learning. Only $75 \%$ of the out of the majority group showed interest in learning the new technologies. This proves that internal barriers including users' perception of technology and their attitude plays a major role in the bigger picture of what is holding the design firms back from adopting VR, AR, and MR technologies for their business and practice. Based on the hierarchy of technology adoption (Hooper and Rieber, 1995) the design consultancy business in the current study was identified with stage 2 of "utilization". Some design practitioners in the firm have already started using VR, AR, and MR in their work --which pushed them through stage 1, familiarization. However, the results of this study confirmed that they are not yet fully depended on new technology where they cannot work without that. Thus, designers have not yet passed the stage 3 of the technology adoption, the "integration". In order to move forward and achieve a higher level within the hierarchy of technology adoption, other than the external upgrade of equipment, technical support, and training, the design firm may consider starting the process of improvement by prioritizing resolving the internal barriers. As part of the effort that helps design practitioners overcome the technology adoption barriers, especially interior barriers, an interview was conducted with a design expert from a large American Midwestern architecture and engineering firm (800 employees), whose designers actively utilize VR, AR, and MR in their design process. It was suggested that the only way to persuade and encourage designers to use immersive technology platforms in their work, is to have them exposed to the technologies as often as possible. Thus, a designated VR station in an open area of office, where everyone can have access to, and be able to observe the process of design in VR on a daily basis, could be a viable option. Otherwise, using technology in private office spaces or conference room for client presentations would not encourage the adoption and active usage of technology. In fact, having the regular exposure to the technologies would trigger the curiosity of designers who may not show interest in using VR, AR, and MR. As a result, they will start asking 
questions, exploring possibilities, and gaining new knowledge about the technologies from their early adapter colleagues (Grammier, 2018).

Based on the result of measuring comfortability of using technology, it can be understood that participants are more comfortable with the types of technologies that are more associated with their traditional design activities such as producing panoramic renderings and walk-through animations. Although, the new technologies such as VR, AR, and MR were less perceived as "comfortable" by participants. This could also be categorized as an internal barrier, where the fear of learning a new technology becomes a challenge for practitioners. This could be described by the model of perceived ease-of-use (Davis, 1989) where the users make a judgment about the amount of effort that is needed for learning the technology. The fear of adopting immersive technologies in a corporate design office could also be the result of the "high-tech syndrome", which leads practitioners to believe that the term technology itself means high technology, thus a coherent view of the technological base of a firm is only for high-tech companies or high-tech designated positions (Ford, 1988). Perhaps one way to resolve this problem could be increasing the employees' exposure to the immersive technologies on a daily basis. By exposure to new hardware, software, and technology, designers may gain more insight of how VR, AR, and MR are utilized in their design work, and become more familiar with how to run the equipment, which enhances their comfortability. Ford (1988) also suggested that managers and business leaders ought to be actively involved with the firm's technology related activities and processes and keep the conversation on technology going within the firm rather than focusing on the output of technology. This may raise the awareness of technology adoption among designers, motivate them to accelerate the adoption process, and get them more comfortable with the new technologies.

Determination of the learning curve exercise showed that there are multiple preferred methods of learning among the participants. Some participants preferred the self-learning option while others preferred the small group training, and one-on-one training sessions. This diversity of learning types may cause issues for business leaders to determine the most effective type of training is for their employees. A similar pattern was identified on the preferred training time. While the majority of participants preferred to spend 2 to 4 hours per week on learning and training, a large group was also interested in spending 4-8 hours a week on learning. Considering the fact that 8 hours embody a full working day, design employees will lose a huge percentage of their weekly working time if the training is included as a required activity. So, the design business will need to come up with a solution to integrate the learning and training in the regular work activities. Despite the convenience of employing the online training programs, this option doesn't seem to be a viable solution due to the workload and productivity priority in the work environment. This exercise proved that the employees' learning curve played a significant role as an external barrier of technology adoption. In fact, it is the factor that along with funding, training method, and technical support. A good practice for design managers and business leaders to create an effective VR, AR, and MR training program for their employees would be focused on adding expert mentors to the design groups. Those who prefer learning the immersive technologies on their own, tend to require less formal assistance from instructors and mentors. However, it does not mean that design firms should not provide support for such individuals. It is important that business leaders provide the essential learning materials and the support for the needs of self-learning as it motivates their designers' self-improvement.

Based on the data collected from the follow up survey and the multiple regression analysis, there was no significant relationship found between attitude toward the adoption of emerging technologies and years of experience of employees as well as their willingness to spend time on learning emerging technologies for design services. This might be an unexpected finding as it is commonly understood that different generations respond to and utilize new immersive technology in immeasurably different ways (Murray, 2011) which results into a perception that a junior employee would have a more open attitude and a higher willingness to learn a new technology than a senior employee. However, the result of this survey has expressed otherwise. This does not mean that it rejects that perception. The data from the follow up survey is collected from one organization within a single design discipline, it cannot be generalized for every group across multiple industries. Yet in this particular case of the study, the difference in years of experience and learning curve does not have any notable effect on the level of immersive technology adoption. 


\section{CONCLUSION}

Immersive technologies such as $\mathrm{VR}, \mathrm{AR}$, and MR have proven to have great impacts on many design professions. Along with the benefits, there are some challenges and barriers that prevent designers and design-centered businesses from adopting and utilizing these technologies. As an answer to the first research question, this present study confirmed that internal and external barriers of technology adoption are applicable to design profession. These barriers embody a pattern that may repeat across many disciplines. Architectural design in this case study is among one of those many design disciplines. These barriers are interrelated, therefore, tackling one barrier at a time may not be the most effective solution for organizations to accelerate the technology adoption. It is crucial to understand their relationship and build strategies that make impacts on the barriers as a whole system. In fact, adopting immersive technologies in organizational level, should be considered as an investment for process improvement rather than a product and technological artifacts for marketing purposes. As a response to the second research question, in this study, learning curve does not appear to have any notable effect on the level of technology adoption. However, the learning curve is a relevant issue to every business when it comes to adopting a new immersive technology. Different businesses will approach this issue differently. However, it is crucial that a transparent conversation on this issue is generated circulated constantly by the business' leadership as a method of developing a healthy working culture and ensuring consistency in the performance of employees.

The present study aimed to identify the challenges of adopting immersive technologies in the design business context. This study may help design firms and creative organizations with providing potential directions on how to overcome the barriers of adopting and utilizing the virtual reality, augmented reality, and mixed reality technologies in their design practice. However, the study has only been able to collect data from the case study of an architectural design office. In future research, a larger sample size of participants and more cases of design businesses is desirable. Also, most participants had a background and expertise in architectural design and engineering. Thus, a diverse participant pool may help improve the generalizability of findings of this study. Expanding the size of participant pool will also help future research utilized demographic data such as gender and age as it may be a predictor of employee's behavior and learning curve. However, such information may be characterized as identifiable data within a medium sized office. Hence this study was limited to only employees' years of experience. Conducting research in different types of design businesses such as product design, urban planning, interior design, or fashion design are encouraged. Future research should not only be limited to specific geographical location and economical structure, a broader collection of businesses from different regions and countries with different economic structure may be conducted to investigate unforeseen barriers of technology adoption in deeper layers. With such conditions, barriers that are results of the organizational culture shall be addressed.

Organizational aspects of technology adoption were laid within the primary topic of this research. However, future studies may tackle the challenges that independent design practitioners such as freelancers are facing. Internal design studios at non-design-oriented corporations may potentially have distinctive agenda using immersive technologies in design for commercial growth. Such studios shall be used as the precedent subjects in future research efforts.

The immersive technologies in this research focused on a suite of media that is represented by VR, AR, and MR. A deeper discussion on each technology from this suite is highly encouraged in future research. Other innovative tools that support design process such as rendering engines, 3D printing technologies, performance simulation and analysis should also have their own focus. Finally, it is crucial to understand how the three-dimensional media can facilitate the dialogue and interaction among designers, stakeholders and clients.

This study was not aimed to provide particular solution to design businesses on how they can tackle these technology adoption barriers. Because, each design business has their own structure, culture, and managerial strategy. However, the outcome of this research may raise the awareness among design managers and practitioners about what may hold them back from technology adoption and utilization 
in their processes. Thus, this study may help them identify their own methods and redraw their strategy to strengthen the future of their design practice.

\section{REFERENCES}

Adam, J.A. (1993), "Virtual reality is for real", IEEE spectrum, Vol 30 No. 10, pp. 22-29. https://dx.doi.org/10.1109/6.237580

Adler, P.S. and Shenbar, A. (1990), "Adapting your technological base: The organizational challenge”, Sloan management review, Vol. 32 No. 1, pp. 25-37. issn: 0019-848X

Azuma, R. T. (1997), “A survey of augmented reality”, Presence: Teleoperators \& Virtual Environments, Vol. 6 No. 4, p. 355. https://dx.doi.org/10.1162/pres.1997.6.4.355

Bereiter, C. (1994), “Constructivism, socioculturalism, and Popper's world 3", Educational researcher, Vol. 23 No. 7, pp. 21-23. https://dx.doi.org/10.3102/0013189X023007021

Berg, L.P. and Vance, J.M. (2017), "Industry use of virtual reality in product design and manufacturing: a survey”, Virtual Reality, Vol. 21 No. 1, pp. 1-17. https://dx.doi.org/10.1007/s10055-016-0293-9

Billinghurst, M., Clark, A., and Lee, G. (2015). A Survey of Augmented Reality. Foundations and Trends ${ }^{\circledR}$ in Human-Computer Interaction, Vol. 8 No. 2-3, pp. 73-272. https://dx.doi.org/10.1561/1100000049

Davis, F. D. (1989), "Perceived usefulness, perceived ease of use, and user acceptance of information technology" MIS quarterly, pp. 319-340. https://dx.doi.org/10.2307/249008

Di Loreto, I., Van Dokkum, L., Gouaich, A. and Laffont, I. (2011, July), "Mixed reality as a means to strengthen post-stroke rehabilitation”. In International Conference on Virtual and Mixed Reality (pp. 11-19). Springer, Berlin, Heidelberg. https://dx.doi.org/10.7717/peerj.469

Ford, D. (1988), "Develop your technology strategy", Long range planning, Vol. 21 No. 5, pp. 85-95. https://dx.doi.org/10.1016/0024-6301(88)90109-4

Frost, P. and Warren, P. (2000), "Virtual reality used in a collaborative architectural design process", In Information Visualization, 2000. Proceedings. IEEE International Conference on. IEEE. (pp. 568-573) https://dx.doi.org/10.1109/iv.2000.859814

Grammier B. (2018), "VR in design process" interviewed by Hoang, D.

Hooper, S. and Rieber, L.P. (1995), “Teaching with technology”, Teaching: Theory into practice, 2013, pp. 154170. https://dx.doi.org/10.1007/978-981-10-0954-9_2

Milgram, P., Takemura, H., Utsumi, A. and Kishino, F. (1995), December. “Augmented reality: A class of displays on the reality-virtuality continuum", In Telemanipulator and telepresence technologies (Vol. 2351, pp. 282-293). International Society for Optics and Photonics. https://dx.doi.org/10.1117/12.197321

Murray, A. (2011), "Mind the gap: Technology, millennial leadership and the cross-generational workforce", The Australian Library Journal, Vol. 60 No. 1, pp. 54-65. https://dx.doi.org/10.1080/00049670.2011.10722556

Nambisan, S. (2002), "Designing virtual customer environments for new product development: Toward a theory", Academy of management review, Vol. 27 No. 3, pp. 392-413. https://dx.doi.org/10.2307/4134386

McCarthy, J. and Wright, P. (2004), “Technology as experience”, Interactions, Vol, 11 No. 5, pp. $42-43$. https://dx.doi.org/10.1145/1015530.1015549

Pontonnier, C., Dumont, G., Samani, A., Madeleine, P. and Badawi, M. (2014), "Designing and evaluating a workstation in real and virtual environment: toward virtual reality based ergonomic design sessions", Journal on Multimodal User Interfaces, Vol. 8 No. 2, pp. 199-208. https://dx.doi.org/10.1007/s12193-0130138-8

Portman, M.E., Natapov, A. and Fisher-Gewirtzman, D. (2015), "To go where no man has gone before: Virtual reality in architecture, landscape architecture and environmental planning. Computers", Environment and Urban Systems, Vol. 54, pp. 376-384. https://dx.doi.org/10.1016/j.compenvurbsys.2015.05.001

Rogers, P.L. (2000), "Barriers to adopting emerging technologies in education", Journal of educational computing research, Vol. 22 No. 4, pp. 455-472. https://dx.doi.org/10.2190/4UJE-B6VW-A30N-MCE5

Schieman, E. and Fiordo, R. (1990), Barriers to Adoption of Instructional Communications Technology in Higher Education. https://dx.doi.org/10.1177/0258042X15572422

Sherman, W.R. and Craig, A.B. (2018), "Understanding virtual reality: Interface, application, and design", Morgan Kaufmann. https://dx.doi.org/10.1145/2792790

Thomas, B. H. (2012), “A survey of visual, mixed, and augmented reality gaming”, Comput. Entertain., Vol. 10 No. 1, pp. 1-33. https://dx.doi.org/10.1145/2381876.2381879

Zyda, M. (2005), "From visual simulation to virtual reality to games", Computer, Vol. 38 No. 9, pp. $25-32$. https://dx.doi.org/10.1109/MC.2005.297 